\title{
Research
}

\section{The relationship between prior antimicrobial prescription and meningitis:}

\author{
a case-control study
}

\begin{abstract}
Background

Recent research into the role of the human microbiome in maintaining health has identified the potentially harmful impact of antimicrobials.
\end{abstract}

\section{Aim}

The association with bacterial and viral meningitis following antimicrobial prescription during the previous year was investigated to determine whether antimicrobials have a deleterious effect on the nasopharyngeal microbiome.

\section{Design and setting}

A case-control study (1:4 cases to controls) was conducted examining the rate of previous antimicrobial exposure in cases of meningitis and in a matched control group. Data from a UK primary care clinical database were analysed using conditional logistic regression.

\section{Results}

A total of 7346 cases of meningitis were identified, 3307 (45\%) viral, 1812 (25\%) bacterial, and $2227(30 \%)$ unspecified. The risks of viral ladjusted odds ratio [AOR] 2.45; 95\% confidence interval $[\mathrm{Cl}]=2.24$ to 2.68 ) or bacterial (AOR 1.98; $95 \% \mathrm{Cl}=1.71$ to 2.30 ) meningitis were both increased following antimicrobial prescription in the preceding year. Patients who received $\geq 4$ antimicrobial prescriptions in the preceding year were at significantly increased risk of all types of meningitis (AOR 2.85; $95 \% \mathrm{Cl}=2.44$ to 3.34 ), bacterial meningitis (AOR 3.06; $95 \% \mathrm{Cl}=2.26$ to 4.15 ) and viral meningitis (AOR 3.23; $95 \% \mathrm{Cl}=2.55$ to 4.08 ) compared to their matched controls.

\section{Conclusion}

There was an increased risk of meningitis following antimicrobial prescription in the previous year. It is possible that this increase was due to an effect of antimicrobials on the microbiome or reflected an increased general susceptibility to infections in these patients.

\section{Keywords}

antimicrobial agents; case-control studies: meningitis; microbiome; primary care.

\section{INTRODUCTION}

The pathogenesis of meningitis is believed to involve microorganisms in the nasopharynx crossing mucosal surfaces into the bloodstream from where they gain access to the subarachnoid space ${ }^{1-8}$ but what triggers this transmission is less clear. One significant risk factor is the presence of Neisseria meningitidis (the causative organism of meningococcal meningitis) in the nasopharyn $x^{9}$; carriage rates as high as $24 \%$ have been identified in 19-year-olds. ${ }^{10,1}$ How is it that a commensal becomes a pathogen? Disturbance of the patient's microbiome, the enormous numbers of commensal, symbiotic, and pathogenic microorganisms that colonise the human body, may be a plausible explanation.

In recent years a number of studies have begun to explore the role of the human microbiome - mainly those commensal bacteria inhabiting the gut - in maintaining health. ${ }^{12,13}$ The integrity of the microbiome may be disrupted by ingesting antimicrobials and there are suggestions that diseases as diverse as asthma and inflammatory bowel disease may have their pathogenesis in antimicrobial-induced harm, particularly in the early years of life. ${ }^{14,15}$ At its most extreme, seen commonly in intensive care units, antimicrobial use is associated with overwhelming infections by resistant organisms due in part to the removal of competition from less resistant bacteria in the patient's gut.

D Armstrong, MSc, PhD, FFPH, FRCGP, professor of medicine and sociology; M Ashworth, DM, MRCP, FRCGP, senior lecturer in general practice; A Dregan, $\mathrm{MSc}, \mathrm{PhD}$, lecturer in translational epidemiology; P White, MD, MRCP, FRCGP, senior lecturer in general practice, Department of Primary Care and Public Health Sciences, King's College London, London.

Address for correspondence

David Armstrong, Department of Primary Care and
The role of excessive antimicrobial prescribing in provoking the emergence of resistant bacteria has now become a major concern ${ }^{16}$ but antimicrobials may also affect the microbiome in more subtle ways. It is possible that an antimicrobial prescription could, ironically in view of its traditional therapeutic role, increase meningitis risk either by damaging commensals in the nasopharynx resulting in decreased competitive inhibition of other organisms or by impairing the protective effect of the immune system. The present study aimed to investigate the possibility that antimicrobial overprescribing may be associated with increased risk of bacterial and viral meningitis. The study also explored a potential dose-response relationship between antimicrobial prescribing and risk of meningitis. Finally, the study assessed the possibility that the risk of meningitis may vary among antimicrobial drugs with broad and narrow spectrum of action.

\section{METHOD}

A case-control study was implemented in a large primary care database, the Clinical Practice Research Datalink (CPRD). CPRD currently contains medical records from 685 general practices representing one of the world's largest electronic databases of anonymised longitudinal data from primary care. The size, patients' characteristics, and geographical distribution of the CPRD primary care practices are representative of

Public Health Sciences, King's College London, Addison House, Guy's Campus, London SE1 1UL, UK E-mail: david.armstrongakcl.ac.uk

Submitted: 2 July 2015; Editor's response: $14 \mathrm{Sep}$ 2015; final acceptance: 29 Sep 2015. (c)British Journal of General Practice This is the full-length article (published online 11 Mar 2016) of an abridged version published in print. Cite this article as: Br J Gen Pract 2016; DOI: 10.3399/bjgp16X684313 


\section{How this fits in}

While there is considerable evidence that antimicrobials are effective against bacterial infection there is very limited evidence that they may trigger or increase the risk of subsequent infections. The finding that meningitis was more common following antimicrobial prescription could be the result of antimicrobials lowering the threshold for later infections whether through disturbance of the patient's microbiome or through other mechanisms. This may be another reason for caution in prescribing antimicrobials.

the UK population. The database includes complete records of all drugs prescribed, clinical diagnoses, referral to consultants, hospitalisations, and investigation results during primary care consultations. All prescriptions are computer generated and are automatically part of the patient medical record. The data have been extensively validated for pharmacoepidemiological, clinical, and health service usage research. ${ }^{17-20}$ Tate and colleagues provide a detailed description of the CPRD. ${ }^{21}$

\section{Study population}

Patients with a recorded diagnosis of meningitis between 1 January 1992 and 31 March 2014 represented the cases. The index date was defined as the first date that a diagnosis of meningitis was recorded. Cases were individually matched with up to four randomly selected controls, on age, sex, family practice, and index date for meningitis lcontrols were given the index date of the meningitis diagnosis of their matched case). Data were extracted in January 2015.

\section{Outcomes}

Medical diagnostic codes were used to identify new diagnoses of meningitis including viral, meningococcal, other bacterial, and unspecified cases.

\section{Exposure}

The exposure variable included any antimicrobial prescription in the 12 months preceding the diagnosis of meningitis; that is, between the baseline and the index date for meningitis. Information extracted included the name of the specific antimicrobial, the number of antimicrobial prescriptions during the follow-up period, and the time lag from drug prescription to meningitis index date. The risk of meningitis following prescription of narrow spectrum antimicrobials and of trimethoprim was also examined: the former could be less harmful to the microbiome and the latter, in targeting predominantly gram-negative urinary tract infections, may have less effect on the mainly gram-positive bacteria in the nasopharyngeal microbiome.

\section{Confounders}

Several variables associated with infection, meningitis risk, and other comorbidities, were included as covariates. These included matching variables lage, sex, index date, and practice); cardiovascular risk factors including cholesterol, body mass index (BMI), (<18.5, 18.5-24.9, 25.0-29.9, 30.034.9 , and $\geq 35 \mathrm{~kg} / \mathrm{m}^{2}$ ), lifestyle factors including smoking (never, ex-smoker, and current smoker); alcohol (never, ex-drinker, and current drinker); C-reactive protein (CRP) levels; comorbidity including depression, cancer, renal disease, and chronic obstructive pulmonary disorders (COPD), cardiovascular diseases lincluding stroke, coronary heart diseases, and diabetes); and co-prescribing including use of antihypertensive drugs, statins, and diabetes treatment. For each confounder, the value closest to the study baseline and before an antimicrobial drug prescription was included. For patients without an antimicrobial drug prescription the value closest to the baseline was selected. Where the closest recorded value for lifestyle and cardiovascular risk factors was $>5$ years prior to the study baseline, a missing value was recorded.

\section{Statistical analysis}

Case-control studies are open to confounding as the apparent relationship between the exposure and disease may be mediated by some other factor. Therefore the relative exposure figures for important clinical factors, such as smoking ${ }^{22}$, that were available in the database and may be associated with both antimicrobial prescription and the occurrence of meningitis adjusted for. In addition, the relationship between antimicrobial consumption and subsequent meningitis may be confounded by indication such that, for example, those patients with prodromal meningitis and/or with a triggering infection could have consulted the GP and obtained an antimicrobial prescription in the days immediately before the emergence of clinically identifiable meningitis. For this reason the time between antimicrobial prescription and meningitis diagnosis was obtained from the database so that the data for the week immediately preceding diagnosis could be treated as possibly being contaminated by a triggering 


\section{Table 1. Baseline characteristics for study cases and controls}

\begin{tabular}{|c|c|c|}
\hline & Cases $(\%), N=7346$ & Controls (\%), N= 29384 \\
\hline Mean age (SD), years & $22(19)$ & $22(19)$ \\
\hline Female & $3773(51)$ & $15092(51)$ \\
\hline \multicolumn{3}{|l|}{ Body mass index ${ }^{a}$} \\
\hline Underweight & $156(2)$ & $730(2)$ \\
\hline Healthy weight & $1422(19)$ & $6113(21)$ \\
\hline Overweight & $795(11)$ & $3030(10)$ \\
\hline Obese & $488(7)$ & $1543(5)$ \\
\hline \multicolumn{3}{|l|}{ Smokinga } \\
\hline Never & $1951(27)$ & $7658(26)$ \\
\hline Past & $417(6)$ & $1497(5)$ \\
\hline Current & $1037(14)$ & $3890(13)$ \\
\hline \multicolumn{3}{|l|}{ Alcohol use $\mathrm{a}^{\mathrm{a}}$} \\
\hline Never & $541(7)$ & 2431 (8) \\
\hline Past & $46(1)$ & $112(0)$ \\
\hline Current & 2156 (29) & $8593(29)$ \\
\hline \multicolumn{3}{|l|}{ Cholesterol, mg/dL } \\
\hline$<5.15$ & $337(5)$ & $1068(4)$ \\
\hline $5.15-6.19$ & 229 (3) & $749(3)$ \\
\hline$\geq 6.20$ & $129(2)$ & $426(1)$ \\
\hline \multicolumn{3}{|l|}{ C-reactive protein, $\mathrm{mg} / \mathrm{L}$} \\
\hline$<1$ & $25(0)$ & $69(0)$ \\
\hline $1-3$ & $119(2)$ & $265(1)$ \\
\hline$>3$ & $239(3)$ & $450(2)$ \\
\hline \multicolumn{3}{|l|}{ Morbidity } \\
\hline Renal disease & $262(4)$ & $615(2)$ \\
\hline Coronary heart disease & $75(1)$ & $238(1)$ \\
\hline Stroke & $61(1)$ & $69(0)$ \\
\hline Diabetes mellitus & $99(1)$ & $286(1)$ \\
\hline Cancer & $85(1)$ & $231(1)$ \\
\hline Depression & $945(13)$ & $2221(8)$ \\
\hline Chronic obstructive pulmonary disease & $1144(16)$ & $3.015(10)$ \\
\hline Chronic inflammation & $196(3)$ & $533(2)$ \\
\hline \multicolumn{3}{|l|}{ Therapy } \\
\hline Lipid lowering & $132(2)$ & $463(2)$ \\
\hline Antihypertensive therapy & $624(8)$ & $1658(6)$ \\
\hline Diabetes mellitus treatment & $95(1)$ & 270 (1) \\
\hline
\end{tabular}

aPercentages do not add up to $100 \%$ as for some patients the data were missing. SD = standard deviation.
Conditional logistic regression was used to determine the adjusted odds ratio (AORs) and $95 \%$ confidence intervals (Cls) for the association between antimicrobial exposure and risk of meningitis. Data on patients were extracted from the study baseline (the later of the start of the patient's record in CPRD or 1 January 1992). Follow-up ended at the earliest of the meningitis index date, date of death, and the end of the CPRD record or 31 March 2014. Analyses estimated the associations between antimicrobial drugs and all-cause and subtypes of meningitis. Separate estimation models were conducted for the association of time lag, number of prescriptions (1, 2, 3, and $\geq 4)$, and drug type with the risk of meningitis. Analyses were adjusted for study confounders and for age, sex, family practice and index date for meningitis by matching. Data for lifestyle and vascular risk factors (such as, BMI, cholesterol, blood pressure, smoking, and drinkingl were not available for all patients and multiple imputation was used to handle missing data. Following Rothman ${ }^{24}$ and Ridker et al ${ }^{25}$ the analyses did not adjust for multiple comparisons. Data were analysed using Stata (version 12).

\section{RESULTS}

A total of 7346 cases of meningitis were identified during the study follow-up; 3307 (45\%) were recorded as viral, 1812 (25\%) as bacterial, and 2227 (30\%) were unspecified. Table 1 shows the baseline characteristics of study cases and controls. Overall, patients with meningitis were more likely to be diagnosed with COPD, depression, and renal diseases compared to their matched controls.

Thirty-five per cent of patients with meningitis had received an antimicrobial prescription in the 12 months preceding their diagnosis compared with $20 \%$ of the control group ladjusted OR [AOR] 2.04, $95 \% \mathrm{Cl}=1.91$ to $2.18, \quad P<0.001)$. Patients with meningitis were more than four times more likely to have had an antimicrobial prescribed in the 7 days preceding the diagnosis (Figure 1).

Figure 1 also shows the AORs for the risk of meningitis of the number of antimicrobial prescriptions recorded in the previous 12 months for each patient divided into the four categories: $1 ; 2 ; 3 ; \geq 4$. Patients prescribed $\geq 4$ antimicrobial prescriptions in the previous 12 months had a higher risk of meningitis (AOR 2.85, 95\% Cl = 2.44 to 3.04 , $P<0.001$ ) compared to those prescribed 0 ( $n=1$ ) or 1 (AOR $1.74,95 \% \mathrm{Cl}=1.62$ to 1.88 ) antimicrobial. 


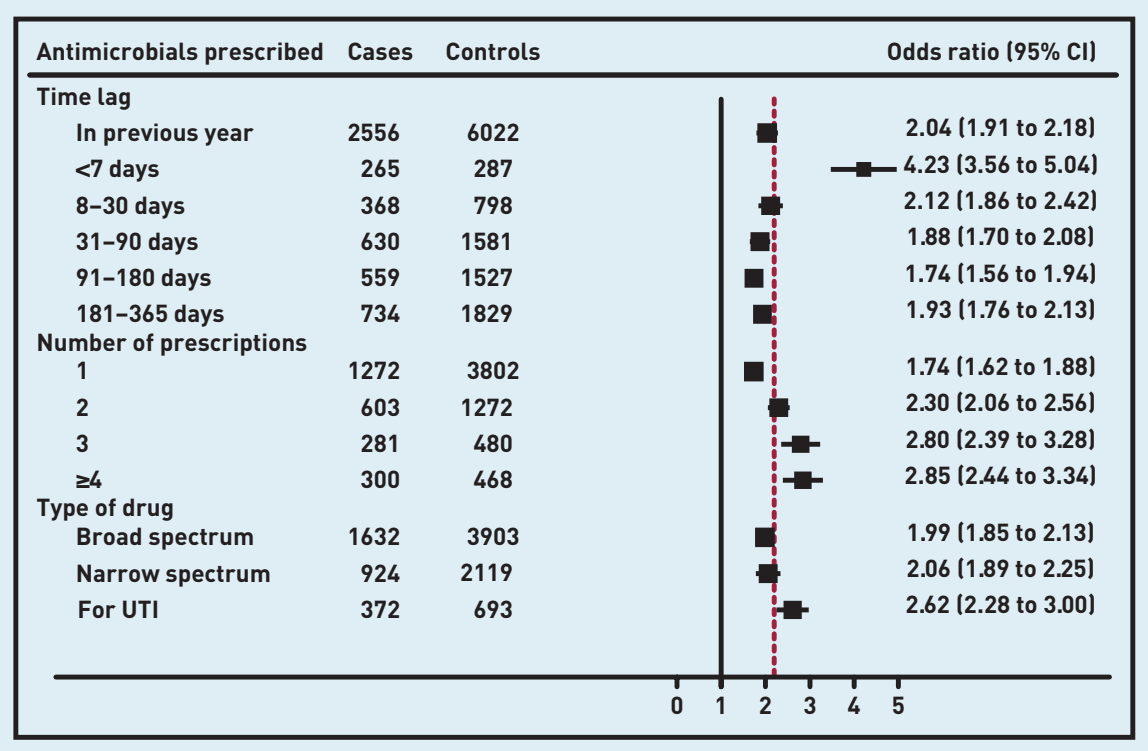

Figure 1. Association between risk of all-cause meningitis and antimicrobials prescription in the previous 12 months. UTI = urinary tract infection.

Figure 2. Association between risk of bacterial meningitis and antimicrobials prescription in the previous 12 months. UTI = urinary tract infection.
The relationships between antimicrobial prescriptions in the previous year land various intervals within that yearl and bacterial and viral meningitis are shown in Figures 2 and 3 respectively. The AORs for both types of meningitis were similar though the risk was higher for viral meningitis if antimicrobials had been prescribed in the 7 days immediately preceding diagnosis (AOR 7.84, 95\% Cl = 6.01 to 10.24, $P<0.001$ ) than for bacterial meningitis (AOR 2.64, $95 \% \mathrm{Cl}=1.86$ to $3.76, P<0.001)$. There were no differences between bacterial and viral meningitis in terms of whether the patient had received a broad or narrow spectrum antimicrobial or trimethoprim prescribed for urinary tract infections.

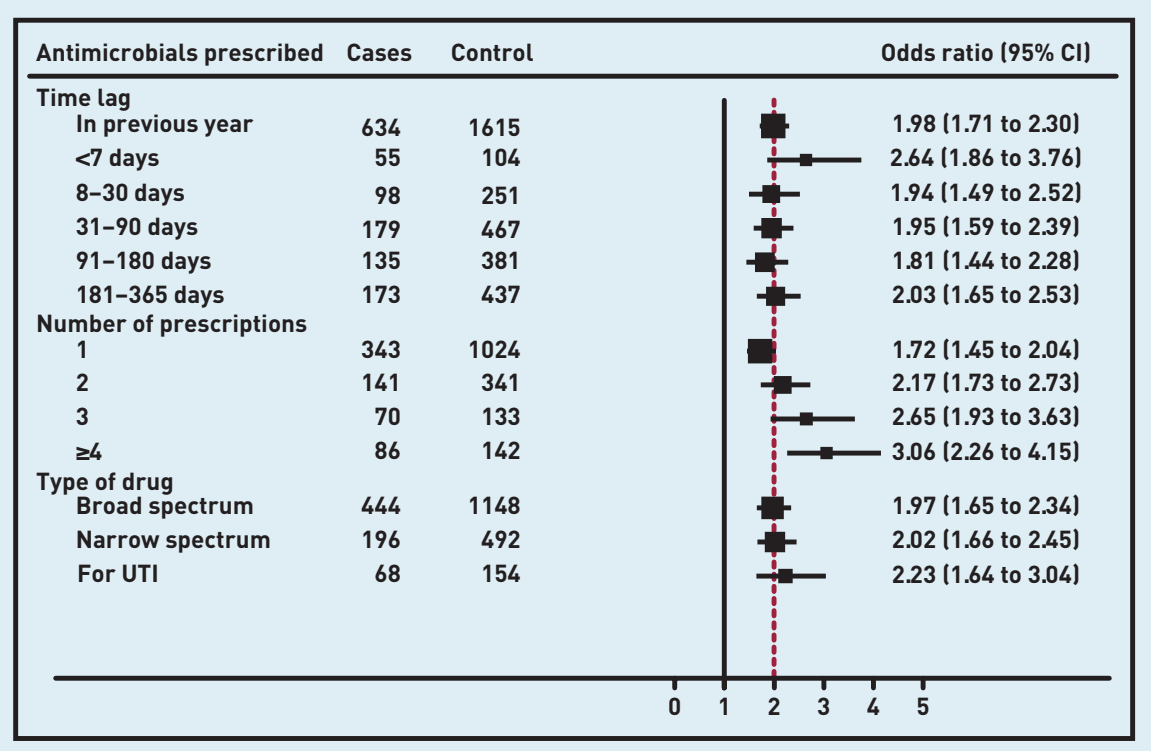




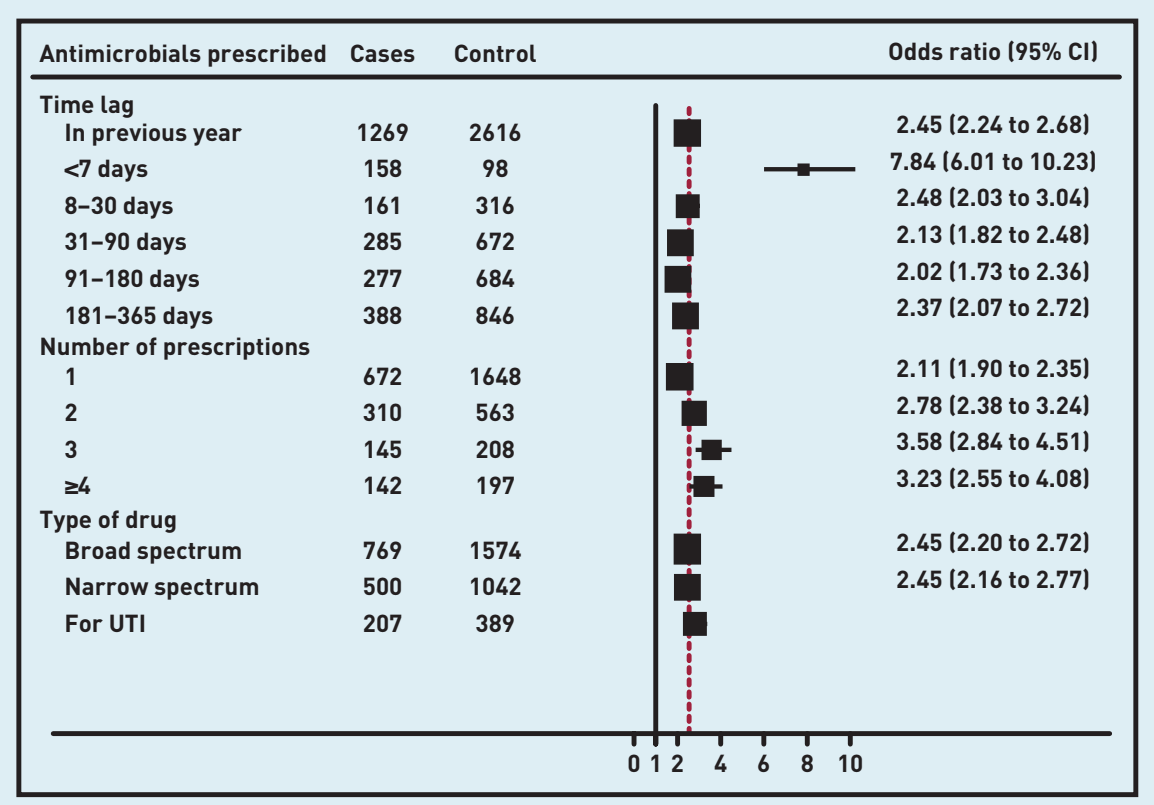

Figure 3. Association between risk of viral meningitis and antimicrobials prescription in the previous 12 months. UTI = urinary tract infection.

\section{Funding}

The research was supported by the National Institute for Health Research (NIHR) Biomedical Research Centre based at Guy's and St Thomas' NHS Foundation Trust and King's College London. The views expressed are those of the author(s) and not necessarily those of the NHS, the NIHR or the Department of Health.

\section{Ethical approval}

Permission to extract and analyse anonymised study data from CPRD was granted by the Independent Scientific Advisory Committee. Formal ethical approval was not required.

\section{Provenance}

Freely submitted; externally peer reviewed.

\section{Competing interests}

The authors have declared no competing interests.

\section{Open access}

This article is Open Access: CC BY-NC 3.0 license (http://creativecommons.org/ licenses/by-nc/3.0/).

Discuss this article

Contribute and read comments about this article: bjgp.org/letters

\section{Strengths and limitations}

The strength of this study lies mainly in the large number of meningitis cases used in the analysis and the likely reliability of the clinical diagnoses and drug prescriptions. The main weakness of this study lies in the difficulty of inferring causality from its case-control design as residual confounding due to unmeasured factors cannot be ruled out. For example, patients with greater general susceptibility to infections, including meningitis, may have presented to their GP more frequently during the previous year and received more antimicrobials. However, the study did adjust for several chronic diseases and related-biomarkers (such as, CRP), commonly used as indicators of frailty.

Meningitis is a diagnosis made in secondary care, most commonly following a lumbar puncture; this diagnosis would then be transcribed into the GP electronic record. It is possible that some diagnoses did not get transcribed into the GP records and/or were transcribed without their specific causal organism being identified. These failures may only minimally impact the present findings. Antimicrobial prescriptions are known to be well recorded in the clinical database lalthough incidental prescriptions given elsewhere, such as in emergency departments or in walk-in centres would not be recorded) but there is no record of whether or not the patients took the prescribed drugs as indicated. Nonadherence by patients, would, if anything, have led to an underestimate of the AORs associated with antimicrobial use.

Nearly $30 \%$ of cases of meningitis were recorded without reference to the specific causative agent. It is possible that correct attribution would alter the size of the estimates observed here, but less so the direction of association. The similarity between viral and bacterial meningitis in their association with previous prescriptions of antimicrobials makes it unlikely that this limitation has made an important difference to the observed relationships. A further weakness of this study is that it was not possible to determine the precise indication for the antimicrobial prescriptions recorded and so it cannot be investigated whether the association between these prescriptions and the diagnosis of meningitis is explained by the indication for the prescription or is independent of that indication. The lack of difference between the classes of antimicrobials examined and their association with meningitis would suggest that the indication was not important.

\section{Comparison with existing literature}

There is evidence that infections in patients who have received antimicrobials in the subsequent 6 months are more likely to be caused by resistant organisms ${ }^{31}$ possibly as a result of decolonisation of antimicrobial sensitive organisms within the microbiome. There is also emerging evidence that disruption of the microbiome in the first year of life has a long-term effect on the risk of diseases such as asthma. ${ }^{32}$

\section{Implications for research and practice}

While the exact mechanism for the association between antimicrobial prescription and subsequent meningitis cannot be determined by the case control design used in this study the size of that association merits further investigation. It also adds another reason for caution in antimicrobial prescribing in general practice to the existing concerns about antimicrobial resistance. 


\section{REFERENCES}

1. Scheld WM, Koedel U, Nathan B. Pathophysiology of bacterial meningitis mechanism(s) of neuronal injury. J Infect Dis 2002; 186: S225-S233.

2. Chadwick DR. Viral meningitis. Br Med Bull 2005; 75-76: 1-14.

3. Tunkel AR, Scheld WM. Pathogenesis and pathophysiology of bacterial meningitis. Clin Microbiol Rev 1993; 6(2): 118-136.

4. Pathan N, Faust SN, Levin M. Pathophysiology of meningococcal meningitis and septicaemia. Arch Dis Child 2003; 88(7): 601-607.

5. Kim KS. Pathogenesis of bacterial meningitis: From bacteraemia to neuronal injury. Nat Rev Neurosci 2003; 4(5): 376-385.

6. Hill DJ, Griffiths NJ, Borodina E, et al. Cellular and molecular biology of Neisseria meningitidis colonization and invasive disease. Clin Sci (Lond)2010; 118(9): $547-564$

7. Quagliarello V. Dissemination of Neisseria meningitidis. N Engl J Med 2011; 364(16): 1573-1575.

8. Coureuil $\mathrm{M}$, Join-Lambert $\mathrm{O}$, Lécuyer $\mathrm{H}$, et al. Mechanism of meningeal invasion by Neisseria meningitidis. Virulence 2012; 3(2): 164-172.

9. Yazdankhah SP, Caugant DA. Neisseria meningitidis: an overview of the carriage state. J Med Microbiol 2004; 53(Pt 9): 821-832.

10. Bogaert D, Hermans PWM, Boelens H, et al. Epidemiology of nasopharyngeal carriage of Neisseria meningitidis in healthy Dutch children. Clin Infect Dis 2005; 40(6): 899-902.

11. Christensen $H$, May M, Bowen $L$, et al. Meningococcal carriage by age: a systematic review and meta-analysis. Lancet Infect Dis 2010; 10(12): 853-861.

12. Human Microbiome Project Consortium. Structure, function and diversity of the healthy human microbiome. Nature 2012; 486(7402): 207-214.

13. Velasquez-Manoff M. Gut microbiome: the peacekeepers. Nature 2015; 518(7540): S3-S11

14. Huang YJ, Boushey HA. The microbiome in asthma. J Allergy Clin Immunol 2015; 135 (1): 25-30

15. Allegretti JR, Hamilton MJ. Restoring the gut microbiome for the treatment of inflammatory bowel diseases. World J Gastroenterol 2014; 20(13): 3468-3474.

16. Davies SC. Annual Report of the Chief Medical Officer, Volume Two, 2011 Infections and the rise of antimicrobial resistance. https://www.gov.uk/ government/uploads/system/uploads/attachment_data/file/138331/CMO_ Annual_Report_Volume_2_2011.pdf (accessed 21 Jan 2016).

17. Dregan A, Moller H, Murray-Thomas T, et al. Validity of cancer diagnosis in a primary care database compared with linked cancer registrations in England. population-based cohort study. Cancer Epidemiol 2012; 36(5): 425-429.
18. Booth HP, Prevost AT, Gulliford MC. Validity of smoking prevalence estimates from primary care electronic health records compared with national population survey data for England, 2007 to 2011. Pharmacoepidemiol Drug Saf2013; 22(12): 1357-1361.

19. Dregan A, Charlton J, Wolfe CD, et al. Is Sodium valproate, an HDAC inhibitor, associated with reduced risk of stroke and myocardial infarction? A nested case-control study. Pharmacoepidemiol Drug Saf 2014; 23(7): 759-767.

20. Charlton RA, Cunnington MC, de Vries CS, et al. Data resources for investigating drug exposure during pregnancy and associated outcomes: the General Practice Research Database (GPRD) as an alternative to pregnancy registries. Drug Saf 2008; 31(1): 39-51.

21. Tate AR, Beloff N, Al-Radwan B, et al. Exploiting the potential of large databases of electronic health records for research using rapid search algorithms and an intuitive query interface. J Am Med Inform Assoc 2014; 21(2): 292-298.

22. Riordan $\mathrm{T}$, Cartwright $\mathrm{K}$, Andrews $\mathrm{N}$, et al. Acquisition and carriage of meningococci in marine commando recruits. Epidemiol Infect 1998; 121(3): 495-505.

23. Hamblin SR, White PA, Tanaka MM. Viral niche construction alters hosts and ecosystems at multiple scales. Trends Ecol Evol 2014; 29(11): 594-549.

24. Rothman KJ. No adjustments are needed for multiple comparisons. Epidemiology 1990; 1(1): 43-46.

25. Ridker PM, Danielson E, Fonseca FA, et al. Rosuvastatin to prevent vascular events in men and women with elevated C-reactive protein. New Eng J Med 2008; 359(21): 2195-2207.

26. Tully J, Viner RM, Coen PG, et al. Risk and protective factors for meningococcal disease in adolescents: matched cohort study. BMJ 2006; 332(7539): 445-450.

27. Hopkins M, Kailasan S, Cohen A, et al. Diversity of environmental singlestranded DNA phages revealed by PCR amplification of the partial major capsid protein. ISME J 2014; 8(10): 2093-2103.

28. Muniesa M, Jofre J. Identifying and analyzing bacteriophages in human fecal samples: what could we discover? Future Microbiol 2014; 9(7): 879-886.

29. Cartwright KA, Jones DM, Smith AJ, et al. Influenza A and meningococcal disease. Lancet 1991; 338(8766): 554-557.

30. Jansen AGSC, Sanders EAM, Van Der Ende A, et al. Invasive pneumococcal and meningococcal disease: association with influenza virus and respiratory syncytial virus activity? Epidemiol Infect 2008; 136(11): 1448-1454.

31. Duffy MA, Hernandez-Santiago V, Orange G, et al. Trimethoprim prescription and subsequent resistance in childhood urinary infection: multilevel modelling analysis. Br J Gen Pract 2013; DOI: 10.3399/bjgp13X665198.

32. Teo SM, Mok D, Pham K, et al. The infant nasopharyngeal microbiome impacts severity of lower respiratory infection and risk of asthma development. Cell Host Microbe 2015; 17(5): 704-715. 